\title{
Relación entre capacidad aeróbica y el nivel de atención en escolares de primaria Relationship between aerobic capacity and level of attention in primary school children
}

\author{
Andres Rosa Guillamón, Eliseo Garcia Canto, Pedro José Carrillo López
}

Universidad de Murcia (España)

\begin{abstract}
Resumen. La capacidad aeróbica constituye el principal exponente de la condición física. La atención es una de las principales funciones cognitivas, directamente relacionada con mecanismos como la percepción, la memoria, el funcionamiento ejecutivo, en procesos como el aprendizaje e incluso en el ajuste psicosocial. El objetivo del presente estudio fue analizar la relación entre capacidad aeróbica y atención selectiva. Es un diseño de estudio descriptivo relacional donde se evaluó la capacidad aeróbica (test de Course-Navette) y la atención (test de Percepción de Semejanzas y Diferencias) en una muestra de 44 escolares (20 mujeres) de 9-10 años. Los escolares con mayor capacidad aeróbica tienen mejores registros en aciertos $(p=0,049)$, omisiones $(p=0,031)$ y control inhibitorio $(p=0,034)$. Los resultados de este trabajo se alinean con la investigación previa que plantea una relación positiva entre capacidad aeróbica y atención. Así, aquellos con mayor capacidad aeróbica presentan una mejor atención selectiva.

Palabras clave: capacidad aeróbica, atención, salud cognitiva, course-navette, escolares.
\end{abstract}

Abstract. Aerobic capacity constitutes the main indicator of physical fitness. Attention is one of the main cognitive functions, directly related to mechanisms such as perception, memory, executive functioning, in processes such as learning or even in psychosocial adjustment.The aim was to analyze the relationship between aerobic capacity and selective attention. The study implemented a descriptive design, evaluating aerobic capacity (Course-Navette test) and selective attention (Perception of Similarities and Differences test) in a sample of 44 students (20 women) between 9-10 years. Students with higher aerobic capacity had better scores in successes $(p=0,049)$, omissions $(p=0,031)$ and inhibitory control $(p=0,034)$. The results of this work are in line with previous research showing a positive relationship between aerobic capacity and attention. In this sense, students with higher aerobic capacity present better selective attention.

Keywords: aerobic capacity, physical activity, attention, health cognitive, course-navette, Schoolchildren.

\section{Introducción}

La evidencia científica disponible indica que la actividad física practicada de manera habitual y en la forma apropiada (sistematizada y de moderada a elevada intensidad,e» 3 METS) constituye una de las mejores estrategias disponibles actualmente para favorecer el bienestar de la población y la salud pública (Castillo-Garzón, 2007; Gálvez et al., 2015; Garber et al., 2011; Rowland, 2007; Torres-Luque, Carpio, Lara \& Zagalaz, 2014; Zurita-Ortega, Ubago-Jiménez, Puertas-Molero, González-Valero, Castro-Sánchez \& Chacón-Cuberos, 2018).

Recientes estudios indican que la actividad física se relaciona de manera inversa con la morbilidad y la mortalidad por enfermedad cardiovascular, entre otras (Lee, Artero, Sui \& Blair, 2010; MartínezGómez, Eisenmann, Gómez-Martínez, Veses, Marcos \& Veiga, 2008). Asimismo, se ha descrito una relación positiva con la calidad de vida (Sloan, Sawada, Martin, Church \& Blair, 2009) y la salud mental (Sui et al., 2009).

A nivel psicológico, constituye uno de los elementos más importantes en el tratamiento coadyuvante en estados de depresión, ansiedad, estrés percibido, nula capacidad de control sobre los impulsos, actitudes antisociales o inactividad y contribuye a mejorar la autoestima, el estado de ánimo y el autoconcepto en adultos y sujetos jóvenes (Babiss \& Gangwisch, 2009; Biddle, Fox \& Boutcher, 2000; Blair, 2009; Dishman et al., 2006; Jiménez, Martínez, Miró \& Sánchez, 2008; Panagiotopoulos et al., 2011).

Los efectos de la actividad física sobre la salud del sistema nervioso central también han sido estudiados en edades tempranas (Tomporowski, Davis, Miller \& Naglieri, 2008; Tomporowski, Lambourne \& Okumura, 2011; Verburgh, Königs, Scherder \& Oosterlaan, 2013); durante la infancia y la adolescencia el cerebro muestra importantes cambios estructurales y funcionales (Martínez-Gómez et al., 2011) y, en este sentido, diversos estudios señalan los efectos beneficiosos que la actividad física tiene sobre el funcionamiento cognitivo en escolares y adolescentes (Hillman, Kamijo \& Scudder, 2011; Monti, Hillman \& Cohen, 2012; Sibley \& Etnier, 2003; Trudeau \& Shephard,2008).

El análisis de la relación entre actividad física y capacidad cognitiva parece tener una mejor interpretación cuando se evalúa el estado de condición física (Pérez-Lobato, Reigal \& Hernández-Mendo, 2016),

Fecha recepción: 02-11-17. Fecha de aceptación: 13-03-18 Eliseo Garcia Canto Eliseo Garcia Canto
eliseo.garcia@um.es entendida como la capacidad del individuo para realizar actividad física (Castillo-Garzón, 2007; Ortega, Ruiz \& Castillo, 2013a). La condición física es un potente marcador biológico del estado general de salud (Ortega et al., 2013a; Ortega, Ruiz, Castillo \& Sjostrom, 2008) y constituye una medida integrada y eficaz de las estructuras y funciones que intervienen en la realización de las actividades humanas. Entre estas funciones, se incluyen la músculo-esquelética, la cardio-respiratoria, la hemato-circulatoria, la endocrino-metabólicay la psico-neurológica(Ruiz et al., 2011).

La capacidad aeróbica (CA) constituye el principal exponente de la condición física (Ortega, Sánchez-López, Solera-Martínez, FernándezSánchez, Sjöströrn \& Martínez-Vizcaíno, 2013b) y refiere a la facultad de un individuo para realizar actividad física de manera prolongada (Gálvez et al., 2015). La CA es un potente indicador fisiológico (predictor desde la infancia) de la salud, especialmente de las funciones cardiovascular, metabólica y respiratoria (Ortega et al., 2013a; Ortega et al., 2008).

Durante los últimos años, se ha incrementado el interés por analizar la relación de la CA con el funcionamiento cognitivo y el rendimiento académico encontrando estudios observacionales (Bass, Brown, Laurson \& Coleman, 2013; Buck, Hillman \& Castelli, 2008; Castelli, Hillman, Buck \& Erwin, 2007; Chaddock et al., 2010; Chaddock, Hillman, Pontifex, Johnson, Raine \& Kramer, 2012; Chaddock, Hillman, Buck \& Cohen, 2011; Hillman, Buck, Themanson, Pontifex \& Castelli, 2009; Hillman, Castelli \& Buck, 2005; Sardinha, Marques, Martins, Palmeira \& Minderico, 2014) y algunas revisiones sistemáticas en escolares y sujetos jóvenes (Conde \& Tercedor, 2015; Rasberry et al., 2011).

El análisis de la atención en el ámbito escolar despierta un gran interés entre científicos, educadores y entrenadores deportivos ya que se trata de una de las principales funciones cognitivas, directamente relacionada con mecanismos como la percepción, la memoria, el funcionamiento ejecutivo y procesos como el aprendizaje, e incluso desempeña un importante papel en el ajuste psicosocial de las personas (Greimel, Wanderer, Rothenberger, Herpertz-Dahlmann, Konrad \& Roessner, 2011; Memmert, Simons \& Grimme, 2009; Tang \& Posner, 2009; Wass, Porayska-Pomsta \& Johnson, 2011).

La atención se define como la capacidad de seleccionar los estímulos que son relevantes para un individuo en una situación concreta (Posner \& Petersen, 1990), en interacción con los sistemas cognitivos, socioemocional y motivacional (Chang \& Burns, 2005). Algunas de sus funciones son mantener el estado de alerta, el equilibrio, la concentración, la orientación y la función ejecutiva, siendo estos procesos funda- 
mentales en el desarrollo madurativo de los niños permitiéndoles controlar sus emociones y pensamientos (de la Vega Marcos, Román, Ruiz, Aguado-Gómez, Hernández \& Sanz, 2015; Gerard, Salicetti, Moncada \& Solano, 2018; Kim, Jiménez-Díaz \& Chen, 2017; Posner, Rothbart, Sheese \& Voelker, 2014).

La atención precede a la percepción y a la acción (Estévez-González, García-Sánchez \& Junqué, 1997), posibilitando la puesta en marcha de otros procesos cognitivos como la memoria y el aprendizaje (Tang, Hölzel \& Posner, 2015), la atención plena (Canales-Lacruz \& Rovira, 2017), el temperamento y la motivación (Chang \& Burns, 2005), siendo un predictor clave en el rendimiento académico (Hackman, Farah \& Meaney, 2010; Batista, Sixto, Honório \& Martins, 2016).

Se distinguen tres tipos de atención: selectiva, dividida y sostenida (García-Ogueta, 2001) las cuales implican redes funcionales distribuidas por la corteza cerebral y estructuras subcorticales conectadas entre sí (Posner \& Petersen, 1990).

Estudios observacionales (Buck, Hillman \& Castelli, 2008; Hillman et al., 2009; Pérez-Lobato et al., 2016; Scudder, Federmeier, Raine, Direito \& Boyd, 2014) y revisiones sistemáticas (Conde \& Tercedor, 2015; Sibley \& Etnier,2003; Maureira \& Flores, 2017) han indagado en la relación entre CA y atención en escolares y adolescentes, observando un rendimiento más eficiente y eficaz en los procesos que requerían control cognitivo, atención selectiva y velocidad de respuesta en aquellos sujetos con una mayor CA. Sin embargo, algunos estudios no han encontrado en escolares una correlación consistente entre la CA y la atención (Maureira, Flores y Veliz, 2015); no observándose efectos positivos sobre la atención en intervenciones basadas en la realización de ejercicio físico aeróbico en las pausas activas de las clases lectivas (Schmidt, Bezing \& Kamer, 2016; Wilson, Olds, Lushington, Petkov, \& Dollman, 2016)

Aunque algunos estudios sugieren una asociación entre condición física y atención selectiva, la naturaleza de este vínculo continúa siendo poco comprendida siendo más frecuente la investigación del fenómeno actividad física y atención, sobre todo en la adultez y en la adolescencia (Pérez-Lobato et al., 2016). Por otro lado, recientes revisiones de la literatura científica indican que, en relación a la temática objeto de estudio, los cuestionarios representan el instrumento más empleado para analizar el nivel de actividad física de las muestras de participantes (Best, 2010; Fedewa \& Ahn, 2011). Ruiz \& Ortega (2009) señalan que, en escolares y adolescentes, los cuestionarios diseñados para valorar la actividad física presentan problemas de fiabilidad y validez, mostrándose la CA como un instrumento más objetivo para valorar el nivel de actividad física y capacidad física. Considerando que una mayor CA presenta beneficios sobre la salud física y psicológica, y la atención selectiva como un proceso fundamental en el funcionamiento cognitivo y en el ajuste psicosocial, la hipótesis de este trabajo fue que los escolares con mejor CA tendrían una mayor habilidad perceptiva y atencional. Por todo ello, el objetivo de esta investigación fue analizar la relación entre CA y atención selectiva en una muestra de escolares.

\section{Material y método}

\section{Participantes}

Se trata de un estudio descriptivo relacional y de corte transversal ex post facto (Thomas \& Nelson, 2007) realizado en 24 varones y 20 mujeres de la Región de Murcia (España), de edades comprendidas entre los 9 y los 10 años (9,5 \pm 0,5 años).

Los participantes fueron seleccionados mediante muestreo no probabilístico intencional (muestra de conveniencia). No fueron incluidos de los grupos naturales de clase cuatro escolares que presentaban alguna patología cardiovascular u osteoarticular aguda o crónica. Antes de aceptar la participación en el estudio, los padres o tutores legales de los escolares así como los responsables del colegio, recibieron información sobre la finalidad y protocolo del mismo; todos dieron su consentimiento informado para que los escolares pudieran participar

La investigación se desarrolló siguiendo las normas deontológicas reconocidas por la Declaración de Helsinki(World Medical Association,
2013) y siguiendo las recomendaciones de Buena Práctica Clínica de la CEE (documento 111/3976/88 de julio de 1990) y la normativa legal vigente española que regula la investigación clínica en humanos (Real Decreto 561/1993 sobre ensayos clínicos). La investigación pertenece a un estudio más amplio que forma parte de una tesis doctoral desarrollada en el Departamento de Expresión Plástica, Musical y Dinámica de la Universidad de Murcia (Murcia, España).

\section{Variables e instrumentos}

LaCAsemidió empleandoel Test de Course-Navette (Léger, Mercier, Gadoury, \& Lambert, 1988). Esta prueba consiste en correr entre dos líneas separadas por 20m en doble sentido, ida y vuelta. El ritmo de carrera es impuesto por una señal sonora. La velocidad inicial es de 8.5 $\mathrm{km} / \mathrm{h}^{-1}$ y se incrementa en $0.5 \mathrm{~km} / \mathrm{h}^{-1}$ con intervalos de un minuto (paliers). El participante debe pisar detrás de la línea de 20 m en el momento justo en que se emite la señal sonora o beep. El test finaliza cuando el participante se detiene porque alcanza la fatiga o cuando por dos veces consecutivas no llega a pisar detrás de la línea al sonido del beep. Se anotó el último palier o medio palier completado. El test se administró una vez. Si el escolar cometió un error de procedimiento se paró y repitió. Para medir esta variable se empleó un equipo audio portátil (Behringer EPA40, Thomann, Burgebrach, Germany) y un dispositivo de memoria USB (Hayabusa, Toshiba, Tokio, Japan). A partir de los resultados obtenidos en el test de Course-Navette y teniendo como referencia los percentiles establecidos para sujetos de 6 a 17 años (Castro-Piñero, Ortega, Keating, González-Montesinos, Sjöstrom, \& Ruiz) los escolares fueron categorizados en dos grupos: menor $\mathrm{CA}\left(\mathrm{mCA},<\mathrm{P}_{50}\right)$ y mayor CA (MCA, e» $\mathrm{P}_{50}$ ). El $\mathrm{P}_{50}$ fue de 2,35. Se estimó indirectamente el consumo máximo de oxígeno ( $\mathrm{VO}_{2}$ máx.) empleando las ecuaciones de Léger: $\mathrm{VO}_{2}$ máx. $=31,025+3,238 * \mathrm{~V}-3,248 * \mathrm{E}+0,1536 * \mathrm{~V} * \mathrm{E}$. En esta, $V$ es la velocidad $\left(\mathrm{km} / \mathrm{h}^{-1}\right)$ de laúltima etapa completa y $E$ es la edad (en años) del sujeto.

La atención selectiva se evaluó mediante el Test de Percepción de Semejanzas y Diferencias (Caras-R) de Thurstone \& Yela (2012). Esta prueba mide la aptitud para percibir, con la mayor velocidad de procesamiento, semejanzas, diferencias y patrones estimulantes parcialmente ordenados. Se utiliza en sujetos de seis a 18 años. Está compuesto por 60 elementos gráficos; cada uno de ellos está formado por tres dibujos esquemáticos de caras con la boca, las cejas y el pelo representados con trazos elementales. En cada conjunto de tres caras, dos son iguales, y la tarea consiste en determinar cuál es la diferente y tacharla. Este test ha sido ampliamente utilizado en el ámbito educativo con la finalidad de evaluar aspectos perceptivos y atencionales en escolares con y sin problemas de atención e hiperactividad. En la realización del test se inhiben las estrategias adoptadas para discriminar entre los distintos ítems. Cuando el sujeto encuentra la cara diferente debe tacharla y seguir con el resto de conjuntos. No existe un orden para cumplimentar el test. El sujeto tiene un tiempo total de tres minutos. La puntuación se obtiene directamente del número total de aciertos, siendo la valoración máxima 60 puntos. Los estudios de fiabilidad de la prueba realizados por Thurstone \& Yela (1985) con individuos a partir de seis años mostraron un coeficiente de fiabilidad de 0.95; en el análisis de validez se observó que la varianza se distribuía principalmente entre los factores de rapidez de percepción e inteligencia espacial.

Esta prueba ha sido correlacionada con otras que valoran la atención e impulsividad como el test DiViSA-UAM o el d2 Test of Attention (Lozano, Capote \& Fernández, 2015), arrojando datos que muestran una fuerte correlación. En el trabajo de Reparaz, Peralta \& Narbona (1996) se calculó la consistencia interna del test obteniendo unos coeficientes de correlación, en cuanto al número de aciertos, por encima de 0.96 en todos los grupos. En otro estudio, Crespo-Eguilaz \& Narbona (2006) se plantearon las siguientes variables: (1) aciertos: número total de respuestas correctas; es considerado como una medida de habilidad perceptiva y atención selectiva; (2) errores: número de respuestas incorrectas; es considerado como una medida de inhibición de respuestas automáticas; (3) control impulsivo o inhibitorio (CI): es considerado como el ratio de la diferencia entre las respuestas correctas e incorrectas, 
dividido entre la suma de correctas e incorrectas (correcta-incorrecta/ correcta+incorrecta). El CI no se tuvo en cuenta en la versión original del test, pero ha sido propuesto por estos autores como una medida del control inhibitorio e impulsividad, ya que se ha encontrado más efectivo que la medición simple del número de respuestas en sujetos con o sin diagnóstico de trastorno por déficit de atención e hiperactividad.

En otra propuesta de interpretación del test, Ison \& Anta (2006) incorporan a la cantidad de estímulos correctamente detectados (aciertos o puntaje directo) que se emplean en la técnica original, los errores y omisiones. El término omisiones hace referencia a las figuras iguales al modelo no señaladas en la tarea, donde (PD) es el puntaje directo, (A) es el número de aciertos, $(\mathrm{E})$ los errores y $(\mathrm{O})$ las omisiones: $\mathrm{PD}=[\mathrm{A}$ - (E $+\mathrm{O})$ ].

Teniendo en cuenta todo esto, en este trabajo se consideraron las siguientes variables: (1) aciertos: número total de respuestas correctas; (2) errores: número de respuestas incorrectas; (3) omisiones: figuras no señaladas en la tarea; y, (4) CI: ratio de la diferencia entre las respuestas correctas e incorrectas, dividido entre la suma de correctas e incorrectas.

\section{Procedimiento}

Se desarrolló un proceso de entrenamiento de los exploradores colaboradores (dos maestros especialistas en educación física). Todos los participantes recibieron las instrucciones para realizar el test de Course-Navette de forma verbal así como en un vídeo de demostración (Ortega, 2011), y se realizó una demostración práctica. Se recomendó que los participantes se abstuvieran de realizar, al menos durante 48 horas, ejercicio físico intenso. Los escolares vistieron ropa deportiva ligera y realizaron un calentamiento de ocho minutos basado en movilidad articular dinámica. La prueba se administró entre las 1000 y las 11'30 horas en grupos de seis escolares en función de la lista de clase. El test de atención fue administrado de forma separada en los dos grupos de escolares. Se utilizó una sala que permitía una separación física suficiente para mantener la privacidad y libertad en la cumplimentación. Se mantuvo la presencia de un investigador para resolver posibles dudas. Los participantes fueron evaluados durante el mes de febrero del curso académico 2016/17.

\section{Análisis estadístico}

Se realizó un análisis descriptivo básico calculando los estadísticos de la media, la desviación estándar, el error estándar y el recuento numérico. La distribución de las variables resultó normal a través de la prueba de Shapiro-Wilk, por lo que las diferencias según sexo ( 0 = varones, 1 = mujeres) se estudiaron mediante un análisis de la covarianza (ANCOVA) ajustado por edad (años) para variables continuas y el test de chicuadrado $\left(X^{2}\right)$ de Pearson para las variables categóricas (sexo yCA). Las diferencias según nivel de CA (mCA y MCA) se estudiaron mediante una prueba ANCOVA ajustada por sexo. La prueba de Levene fue utilizada para comprobar la homogeneidad de las varianzas. Secalculóla $c^{2}$ para estimar el tamaño del efecto y la potencia estadística (á). Los datos fueron analizados con el programa Statistical Package for the Social Sciences (v.20.0 de SPSS Inc., Chicago, Illinois, EE.UU.).

\section{Resultados}

La tabla 1 muestra los resultados arrojados por el análisis descriptivo de los parámetros de CA y atención así como el análisis de las diferencias según sexo. La prueba ANCOVA detectó diferencias estadísticamente significativas en Course-Navette $(p=0.006)$, velocidad de carrera $(p=0.025)$ y $\mathrm{VO}_{2}$ máx $(p=0.025)$. El test de chicuadrado arrojó diferencias estadísticamente significativas en el nivel de $\mathrm{CA}(p=0.008)$. No se observaron diferencias estadísticamente significativas en la atención.

Como análisis complementario y con el propósito de verificar si la CA se correlacionaba con la atención se llevó a cabo la prueba de correlaciones parciales controladas por sexo. En la tabla 2 se aprecia que el cociente $r$ de Pearson se incrementaba en aciertos $(p=0.048)$ y CI $(p=$ 0.027) y disminuía en omisiones $(p=0.028)$ con un mejor rendimiento
Tabla 1.

Descriptivos básicos y diferencias según sexo.

\begin{tabular}{|c|c|c|c|c|c|c|c|}
\hline & Varones $(n=24)$ & & Mujeres $(n=20)$ & & & & \\
\hline & $\mathrm{M} \pm \mathrm{DE}$ & $\mathrm{EE}$ & $\mathrm{M} \pm \mathrm{DE}$ & $\mathrm{EE}$ & $p$ valor & $?^{2}$ & $\mathrm{a}$ \\
\hline $\begin{array}{l}\text { Aciertos } \\
\text { IC (95\%) }\end{array}$ & $\begin{array}{c}27.2 \pm 6.9 \\
(24.4-29.9)\end{array}$ & 1,3 & $\begin{array}{c}26.6 \pm 6.2 \\
(23.5-29.5)\end{array}$ & 1.4 & .747 & .003 & .062 \\
\hline $\begin{array}{l}\text { Errores } \\
\text { IC (95\%) }\end{array}$ & $\begin{array}{l}4.5 \pm 3.4 \\
(3.1-5.7)\end{array}$ & 0,6 & $\begin{array}{l}4.5 \pm 2.7 \\
(3.0-5.8)\end{array}$ & .7 & 993 & .001 & .050 \\
\hline $\begin{array}{l}\text { Omisiones } \\
\text { IC (95\%) }\end{array}$ & $\begin{array}{l}28.5 \pm 8.9 \\
(25.0-31.9)\end{array}$ & 1,7 & $\begin{array}{c}29.0 \pm 7.3 \\
(25.2-32.7)\end{array}$ & 1.8 & .836 & .001 & .055 \\
\hline $\begin{array}{l}\text { Control ínhibitorio } \\
\text { IC (95\%) }\end{array}$ & $\begin{array}{c}31.5 \pm 8.7 \\
(28.1-32.8)\end{array}$ & 1,6 & $\begin{array}{c}30.8 \pm 7.3 \\
(27.1-34.5)\end{array}$ & 1.8 & .795 & .002 & .058 \\
\hline $\begin{array}{l}\text { C-N (paliers) } \\
\text { IC (95\%) }\end{array}$ & $\begin{array}{l}3.0 \pm 1.3 \\
(2.6-3.4)\end{array}$ & 0,2 & $\begin{array}{c}2.2 \pm .6 \\
(1.6-2.6)\end{array}$ & .2 & .006 & .172 & .814 \\
\hline $\begin{array}{l}\text { VC ( ( km/h-1) } \\
\text { IC (95\%) }\end{array}$ & $\begin{array}{c}9.77 \pm 0.77 \\
(9.5-10.0)\end{array}$ & 0,1 & $\begin{array}{c}9.35 \pm 0.33 \\
(9.0-9.6)\end{array}$ & .1 & .025 & .116 & .622 \\
\hline $\begin{array}{l}\mathrm{VO}_{2} \operatorname{máx}\left(\mathrm{ml} / \mathrm{kg} / \mathrm{min}^{-1}\right) \\
\text { IC (95\%) }\end{array}$ & $\begin{array}{c}46.2 \pm 3.5 \\
(44.9-47.3)\end{array}$ & 0,6 & $\begin{array}{c}44.1 \pm 1.9 \\
(42.8-45.4)\end{array}$ & .6 & .025 & .117 & .622 \\
\hline & $n(\%)$ & & $n(\%)$ & & $? 2$ & $p$ va & \\
\hline & & & $18(60.0$ & & 1.200 & & \\
\hline & $12(85$ & & $2(14.3)$ & & 7.143 & & \\
\hline
\end{tabular}

$\mathrm{M} \pm \mathrm{DE}=$ media \pm desviación estándar; $\mathrm{EE}=$ error estándar; $\mathrm{IC}=$ intervalo de confianza; ? ${ }^{2}=$ tamaño del efecto; a = potencia estadística; $\mathrm{CN}$ = Course-Navette; $\mathrm{VC}$ = velocidad de carrera; $\mathrm{VO}_{2}$ máx = consumo máximo de oxígeno; $\mathrm{mCA}=$ menor capacidad aeróbica; $\mathrm{MCA}=$ mayo capacidad aeróbica.

Tabla 2.

Correlaciones entre capacidad aeróbica (paliers) y atención.

\begin{tabular}{llrrrc}
\multicolumn{6}{c}{ Correlaciones entre capacidad aeróbica (paliers) y atención. } \\
\hline \multicolumn{7}{c}{ C-N (paliers) } & \multicolumn{1}{c}{ Aciertos } & Errores & Omisiones & Control inhibitorio \\
& $p$ valorson & .300 & .232 & -.330 & .333 \\
& .048 & .130 & .028 & .027 \\
\hline
\end{tabular}
$\frac{p \text { valo }}{\mathrm{CN}=\text { Course-Navette. }}$

Tabla 3.

\begin{tabular}{|c|c|c|c|c|c|c|c|}
\hline & MCA $(n=30)$ & & $\operatorname{MCA}(\mathrm{n}=14)$ & & & & \\
\hline & $\mathrm{M} \pm \mathrm{DE}$ & $\mathrm{EE}$ & $\mathrm{M} \pm \mathrm{DE}$ & $\mathrm{EE}$ & $p$ valor & $?^{2}$ & $\mathrm{a}$ \\
\hline $\begin{array}{l}\text { Aciertos } \\
\text { IC (95\%) }\end{array}$ & $\begin{array}{c}25,6 \pm 5,7 \\
(22,9-27,8)\end{array}$ & 1.2 & $\begin{array}{c}29,6 \pm 7,5 \\
(26,3-27,7)\end{array}$ & 1.8 & .049 & .091 & .507 \\
\hline $\begin{array}{l}\text { Errores } \\
\text { IC (95\%) }\end{array}$ & $\begin{array}{c}4,1 \pm 2,5 \\
(2,8-5,1)\end{array}$ & .6 & $\begin{array}{l}5,3 \pm 4,0 \\
(3,7-7,2)\end{array}$ & .9 & .183 & .043 & .262 \\
\hline $\begin{array}{l}\text { Omisiones } \\
\text { IC (95\%) }\end{array}$ & $\begin{array}{c}30,4 \pm 6,6 \\
(27,7-33,7)\end{array}$ & 1.5 & $\begin{array}{r}25,1 \pm 10,0 \\
(19,9-28,9)\end{array}$ & 2.2 & .031 & .109 & .590 \\
\hline $\begin{array}{l}\text { Control inhibitorio } \\
\text { IC }(95 \%)\end{array}$ & $\begin{array}{c}29,5 \pm 6,4 \\
(26,3-32,2)\end{array}$ & 1.4 & $\begin{array}{c}34,8 \pm 9,9 \\
(30,8-39,8)\end{array}$ & 2.2 & .034 & .105 & .573 \\
\hline
\end{tabular}
capacidad aeróbica.

en el test de Course-Navette.

$\mathrm{Al}$ dividir la muestra según el nivel de CA (mCA y MCA), la prueba ANCOVA arrojó diferencias estadísticamente significativas en aciertos ( $p=0.049)$, errores $(p=0.031)$ y CI $(p=0.034)$ (tabla 3$)$.

\section{Discusión}

El objetivo de esta investigación fue analizar la relación entre CA y atención selectiva en una muestra de escolares de 9-10 años. Al dividir la muestra según sexo, se observó que no existían diferencias estadísticamente significativas en la atención selectiva. En el rendimiento físico, se encontró que los varones presentaban un mayor nivel de CA (tabla 1), lo que concuerda con lo establecido en la literatura científica (Gálvez et al., 2015; Gálvez et al., 2016; Ortega et al., 2013; Ortega et al., 2008; Ortega et al., 2013b). Estas diferencias podrían deberse a la frecuencia de actividad física ya que aquellos grupos más activos tienen una mayor capacidad cardiorrespiratoria (Torres-Luque et al., 2014). Rowland (2007) atribuye estas diferencias a que los varones tienen mayores valores deVO $\mathrm{O}_{2}$ máx. en términos absolutos que las mujeres.

Los resultados de este trabajo han puesto de manifiesto que la CA se asocia positivamente con la atención selectiva, lo que demuestra la hipótesis del estudio. Cuando se categorizó a los escolares según el rendimiento físico en el test de Course-Navette se observó que aquellos que tenían una MCA presentaban mejores registros en aciertos ( $p=$ 0.049 ), omisiones ( $p=0.031), \mathrm{y} \mathrm{CI}(p=0.034)$ que sus pares homólogos con mCA (tabla 3).

Aunque algunos estudios sugieren una fuerte asociación entre CA y atención, los mecanismos que intervienen en este vínculo continúan siendo poco comprendidos. No obstante, en base a lo establecido en la literatura científica estos resultados pueden ser debidos a que en escolares niveles más elevados de CA están asociados con mayores volúmenes del hipocampo y de los ganglios basales, con un desempeño superior en las tareas en las que es primordial el control de la atención e interferencia, y con mejores índices de potencial cerebral relacionados con eventos elevados de la función ejecutiva (Chaddock et al., 2010). 
Asimismo, estudios como el diseñado por Hillman et al. (2005) sugieren que la CA podría estar asociada con el aumento de la activación neuroeléctrica relacionada con la asignación de los recursos de atención y memoria de trabajo, lo que tal vez podría influir en la velocidad de procesamiento, respuesta y control de la conducta en los escolares.

Los resultados de este trabajo se muestran coherentes con los hallazgos observados en la literatura científica en escolares (Buck et al., 2008; Pontifex et al., 2011; Scudder et al., 2014), adolescentes (PérezLobato et al., 2016) y adultos (Barnes, Yaffe, Satariano \& Tager, 2003). Además, estos hallazgos podrían ser coherentes con los encontrados en aquellos trabajos que sugieren que la CA es el parámetro de la condición física que muestra una mayor interrelación con la salud mental (Gálvez et al., 2015; Gálvez et al., 2016), siendo la variable que mejor predice el funcionamiento cognitivo (Buck et al., 2008; Fedewa \& Ahn, 2011; Hillman et al., 2009; Pérez-Lobato et al., 2016; Pontifex et al., 2011; Tomporowski et al., 2008), aunque no coincide con los hallazgos reportados en el estudio transversal de Maureira et al. (2015), que mostraron que la CA estimada a partir del $\mathrm{VO}_{2}$ máx. no se correlacionaba ni con la atención ni con el rendimiento académico en escolares chilenos de 10 a 13 años. En el mismo sentido, recientes investigaciones longitudinales concluyen que intervenciones de tres a cuatro semanas de duración con sesiones basadas en la realización de 10 minutos de ejercicio físico cardiovascular no tienen efecto inmediato sobre la atención selectiva y mantenida en escolares de enseñanza primaria (Schmidt et al., 2016; Wilson et al., 2016).

Los resultados de este trabajo han puesto de relieve un comportamiento similar en todas las medidas del test de atención. Los escolares con MCA obtuvieron mejores registros en aciertos, omisiones y CI, lo que coincide con lo establecido en otros trabajos que indican una relación positiva de la CA con la atención selectiva y la velocidad para procesar la información (Buck et al., 2008; Guiney \& Machado, 2013). En la misma línea que Hillman et al. (2009) y Pontifex et al. (2011), los escolares con MCA mostraron un mejor CI. No obstante, las diferencias no fueron significativas en errores, no pudiéndose extraer conclusiones sólidas, tal y como también indican Pérez-Lobato et al. (2016).

Teniendo en cuenta la relación positiva establecida en la literatura científica entre la actividad física y la condición física con el funcionamiento cognitivo (Best, 2010; Chaddock et al., 2010; Chaddock et al., 2011; Chaddock et al., 2012; Hillman et al., 2011; Monti et al., 2012; Scudder et al., 2014; Sibley \& Etnier, 2003; Tomporowski et al., 2011), y con la atención selectiva (Guiney \& Machado, 2013; Trudeau \& Shephard, 2008), se puede considerar que los resultados de este trabajo se aproximan a los de otros estudios que habían indicado relaciones positivas entre CA y capacidad cognitiva en escolares y adolescentes (Buck et al., 2008; Hillman et al., 2009; Pontifex et al., 2011) y entre CA y rendimiento académico (Bass, Brown, Laurson \& Coleman, 2013; Castelli, Hillman, Buck \& Erwin, 2007; Sardinha, Marques, Martins, Palmeira \& Minderico, 2014).

En este sentido, se ha descrito que la actividad física de elevada intensidad (e» 6 METS) en escolares podría ser un medio eficaz para influir en este fenómeno a través del incremento de la aptitud cardiorrespiratoria (Pérez-Lobato et al., 2016). En la misma línea, Hötting \& Röder (2013) tras llevar a cabo una revisión de la evidencia científica sobre los efectos del ejercicio físico aeróbico y de fuerza muscular sobre la conducta atencional, sugieren que para que perduren los beneficios neurocognitivos inducidos se debe incrementar el nivel de fitness cardiovascular

Los hallazgos de este trabajo podrían ser de interés para la comunidad educativa ya que contribuyen a fomentar cambios en el currículo académico dado el aumento actual en el comportamiento sedentario y el bajo nivel de CA en estos grupos de edad (Gálvez et al., 2015; Gálvez et al., 2016; Ortega et al., 2013a; Ortega et al., 2008; Ortega et al., 2013b; Verburgh et al., 2013). Por ello, se debe promover un estilo de vida físicamente activo en los escolares (Chaddock et al., 2012) con el fin de favorecer el desarrollo de la CA y preservar la salud cognitiva presente y futura, ya que los beneficios cognitivos asociados con una mayor aptitud aeróbica en escolares pueden tener implicaciones importantes para el aprendizaje y el rendimiento académico (Scudder et al., 2014).

Futuros estudios longitudinales o cuasiexperimentales pueden tener en cuenta las limitaciones de este trabajo, que refieren sobre todo a su diseño transversal, al tamaño y al tipo de selección de la muestra, que impide la determinación de una evolución a largo plazo del problema de estudio, así como el establecimiento de una relación de causalidad entre las variables analizadas. Para aportar un mayor conocimiento científico sería importante considerar variables como el estatus sociocultural, la actividad física, los hábitos de descanso, sueño y comidas o la personalidad, entre otros.

En conclusión, los resultados de este trabajo realizado con escolares de 9-10 años se alinean con la investigación previa que plantea una relación positiva entre CA y atención. Así, aquellos con MCA presentan una mejor atención selectiva.

\section{Referencias}

Babiss, L. A., \& Gangwisch, J. E. (2009). Sports participation as a protective factor against depression and suicidal ideation in adolescents as mediated by self-esteem and social support.Journal of Developmental and Behavioral Pediatrics, 30(5), 376-384.

Barnes, D. E., Yaffe, K., Satariano, W. A., \& Tager, I. B. (2003). A longitudinal study of cardiorespiratory fitness and cognitive function in healthy older adults. Journal of the American Geriatrics Society, 51(4), 459-465.

Bass, R. W., Brown, D.D., Laurson, K. R., \& Coleman, M. M. (2013). Physical fitness and academic performance in middle school students. Acta Paediatrica, 102, 832-837.

Batista, M., Sixto, D., Honório, S. \& Martins, J. (2016). The practice of physical activity related to selfesteem and academical performance in students of basic education. Journal of Human Sport \& Exercise, 11(2), 297-310.

Best, J. R. (2010). Effects of physical activity on children's executive function: Contributions of experimental research on aerobic exercise. Developmental Review, 30, 331-351.

Biddle, S., Fox, K. R., \& Boutcher, S. H. (2000). Physical activity and psychological well-being. Psychology Press.

Blair, S. N. (2009). Physical inactivity: the biggest public health problem of the 21st century. British Journal of Sports Medicine, 43(1), 1-2.

Buck, S. M., Hillman, C. H., \& Castelli, D. M. (2008).The relation of aerobic fitness to stroop task performance in preadolescent children. Medicine and Science in Sports and Exercise, 40, 166-172.

Canales-Lacruz, I. \& Rovira, G. (2017). Mindfulness Practices in University Students. Perception of Positive and Negative Aspects. Retos, 31, 118-122.

Castelli, D. M., Hillman, C. H., Buck, S. M., \& Erwin, H. (2007). Physical fitness and academic achievement in 3rd and 5th grade students. Journal of Sport \& Exercise Psychology, 29, 239-252.

Castro-Piñero, J., Ortega, F. B., Keating, X.D., González-Montesinos, J. L., Sjöstrom, M., \& Ruiz, J. R. (2011). Percentile values for aerobic performance running/walking field tests in children aged 6 to 17 years; influence of weight status. Nutrición Hospitalaria, 26, 572-578.

Chaddock, L., Erickson, K. I., Prakash, R. S., Kim, J. S., Voss, M. W., Van Patter, M.,... Kramer,A. F.(2010). Aneuroimaging investigation of the association between aerobic fitness, hippocampal volume and memory performance in preadolescent children. Brain Research, 1358, 172-183.

Chaddock, L., Hillman, C. H., Buck, S. M., \& Cohen, N. J. (2011). Aerobic fitness and executive control of relational memory in preadolescent children. Medicine \& Science in Sports \& Exercise, 43, 344-349.

Chaddock, L., Hillman, C. H., Pontifex, M. B., Johnson, C. R., Raine, L. B., \& Kramer, A. F. (2012). Childhood aerobic fitness predicts cognitive performance one year later. Journal of sports sciences, 30(5), 421-430.

Castillo-Garzón, M.J. (2007). Physical fitness is an important 
contributor to health for the adults of tomorrow. Selección, 17(1), 2-8.

Chang, F., \& Burns, B. M. (2005). Attention in preschoolers: Associations with effortful control and motivation. Child Development, 76(1), 247-263.

Conde, M.A., \& Tercedor, P. (2015). Physical activity, physical education and ûtness could be related to academic and cognitive performance in young. A systematic review. Arch Med Deporte, 32(2), 100-109.

Crespo-Eguilaz, N., \& Narbona, J. (2006). Subtipos de trastorno específico del desarrollo del lenguaje: perfiles clínicos en una muestra hispanohablante. Revista de Neurología, 43, 193-200.

de la Vega Marcos, R., Román Tabanera, M., Ruiz Barquín, R., AguadoGómez, R., Hernández, J.M. \& Sanz Serrano,A. (2015). Attentional Training Analysis by Videoconsole. Revista Internacional de Medicina y Ciencias de la Actividad Física y el Deporte, 15(58), 339353.

Dishman, R. K., Hales, D. P., Pfeiffer, K. A., Felton, G., Saunders, R., Ward, D. S., \& Pate, R. R. (2006). Physical self-concept and selfesteem mediate cross-sectional relations of physical activity and sport participation with depression symptoms among adolescent girls. Health Psychology, 25(3), 396-407.

Estévez-González, A., García-Sánchez, C., \& Junqué, C. (1997). La atención: una compleja función cerebral. Revista de neurología, 25(148), 1989-1997.

Fedewa, A. L., \& Ahn, S. (2011). The effects of physical activity and physical fitness on children's achievement and cognitive outcomes: a meta-analysis. Research Quarterly for Exercise and Sport, 82, 521-535.

Gálvez, A., Rodríguez-García, P.L., García-Canto, E., Rosa, A., Pérez Soto, J.J., Tárraga, M.L., \& Tárraga, P.J. (2015). Capacidad aeróbica y calidad de vida en escolares de 8 a 12 años. Clínica e Investigación en Arteriosclerosis, 27(5), 239-245.

Gálvez, A., Rodríguez-García, P.L., Rosa, A., García-Cantó, E., PérezSoto, J.J., Tárraga, P.J., \& Tárraga, M.L. (2016). Capacidad aeróbica, estado de peso y autoconcepto en escolares de primaria. Clínica e Investigación en Arteriosclerosis, 28(1), 1-8.

Garber, C.E., Blissmer, B., Deschenes, M.R., Franklin, B.A., Lamonte, M.J., Lee, I.M.,... Swain, D.P.(2011). American College of Sports Medicine position stand. Quantity and quality of exercise for developing and maintaining cardiorespiratory, musculoskeletal, and neuromotor fitness in apparently healthy adults: guidance for prescribing exercise. Med Sci Sports Exerc, 43(7), 1334-59.

García-Ogueta, M. I. (2001). Mecanismos atencionales y síndromes neuropsicológicos. Rev Neurol., 32(5), 463-467.

Gerard, Salicetti, Moncada, \& Solano (2018). Balance, attention and concentration improvements following an exergame training program in elderly. Retos, 33, 102-105.

Greimel, E., Wanderer, S., Rothenberger, A., Herpertz-Dahlmann, B., Konrad, K., \& Roessner, V. (2011). Attentional performance in children and adolescents with tic disorder and co-occurring attentiondeficit/hyperactivity disorder: New insights from a 2x2 factorial design study. Journal of Abnormal Child Psychology, 39, 819-828.

Guiney, H., \& Machado, L. (2013).Benefits of regular aerobic exercise for executive functioning in healthy populations. Psychonomic Bulletin \& Review, 20(1), 73-86.

Hackman, D. A., Farah, M. J., \& Meaney, M. J. (2010). Socioeconomic status and the brain: mechanistic insights from human and animal research. Nature reviews. Neuroscience, 11(9), 651.

Hillman, C. H., Buck, S. M., Themanson, J. R., Pontifex, M. B., \& Castelli, D. (2009). Aerobic fitness and cognitive development: Event-related brain potential and task performance indices of executive control in preadolescent children. Developmental Psychology, 45, 14-129.

Hillman, C. H., Castelli, D. M., \& Buck, S. M. (2005). Aerobic fitness and neurocognitive function in healthy preadolescent children. Medicine and science in sports and exercise, 37(11), 1967.

Hillman, C.H., Kamijo, K., \& Scudder, M. (2011). Areview of chronic and acute physical activity participation on neuroelectric measures of brain health and cognition during childhood. Preventive Medicine, 52(Suppl 1), S21-S28.

Hötting, K., \& Röder, B. (2013). Beneficial effects of physical exercise on neuroplasticity and cognition. Neuroscience \& Biobehavioral Reviews, 37(9), 2243-2257.

Ison, M. S., \& Anta, F. G. (2006). Estudio normativo del test de Percepción de diferencias (caras) en niños mendocinos. Interdisciplinaria, 23(2), 203-231.

Jiménez, M. G., Martínez, P., Miró, E., \& Sánchez, A. I. (2008). Bienestar psicológico y hábitos saludables: ¿están asociados a la práctica de ejercicio físico? International Journal of Clinical and Health Psychology, 8(1), 185-202.

Kim, T., Jiménez-Díaz, J., \& Chen, J. (2017). The effect of attentional focus in balancing tasks: A systematic review with meta-analysis. Journal of Human Sport and Exercise, 12(2), 463-479.

Lee, D.C., Artero, E.G., Sui, X., \& Blair, S.N (2010). Mortality trends in the general population: the importance of cardiorespiratory fitness. J Psychopharmacol, 24(4 Suppl), 27-35.

Léger, L. A., Mercier, D., Gadoury, C., \& Lambert, J. (1988).The multistage 20 metre shuttle run test for aerobic fitness. Journal of Sports Sciences, 6(2), 93-101.

Lozano, J. H., Capote, E., \& Fernández, M. P. (2015). Validez convergente de las medidas de atención e impulsividad del Test de Discriminación Visual Simple de Árboles (DiViSA-UAM). Anales de Psicología, 31(1), 74-83.

Martínez-Gómez, D., Eisenmann, J.C., Gómez-Martínez, S., Veses, A., Marcos, A., \& Veiga, O.L. (2008). Sedentarismo, adiposidad y factores de riesgo cardiovascular en adolescentes. EstudioAFINOS. Rev Esp Cardiol, 63(3), 277-85.

Martínez-Gómez, D., Ruiz, J.R., Gómez-Martínez, S., Chillón, P., Rey-López, J.P., Díaz, L.E., Castillo, R., Veiga, O. L., \& Marcos, A. (2011). Active commuting to school and cognitive performance in adolescents: The AVENA study. Arch Pediatr Adolesc Med, 165,300-5.

Maureira, F., \& Flores, E. (2017). Efectos del ejercicio físico sobre la atención: una revisión de los últimos años. Revista de Ciencias de la Actividad Física UCM, 18(1), 73-83.

Memmert, D., Simons, D. J., \& Grimme, T. (2009). The relationship between visual attention and expertise in sports. Psychology of Sport and Exercise, 10, 146-151.

Monti, J. M., Hillman, C. H., \& Cohen, N. J. (2012). Aerobic fitness enhances relational memory in preadolescent children: The FITKids randomized control trial. Hippocampus, 22, 1876-1882.

Ortega, F. B. (2011, 8 de abril). ALPHA-FIT battery for children and adolescents: 20 m Shuttle Run Test.wmv [Archivo de video]. Recuperado de http://www.youtube.com/watch?v=Fg7Suqa46hU.

Ortega, F. B., Ruiz, J., \& Castillo, M. J. (2013a). Physical activity, physical ûtness, and overweight in children and adolescents: evidence from epidemiologic studies. Endocrinología y Nutrición, 60(8), 458-469.

Ortega, F.B., Ruiz, J.R., Castillo, M.J., \& Sjöström, M. (2008). Physical fitness in childhood and adolescence: a powerful marker of health. Int J Obes (Lond), 32, 1-11.

Ortega, F.B., Sánchez-López, M., Solera-Martínez, M., FernándezSánchez, A., Sjöströrn, M., \& Martínez-Vizcaíno, V. (2013b). Selfreported and measured cardiorespiratory fitness similarly predict cardiovascular disease risk in Young adults. Scand J Med Sci Sports 23(6), 749-57.

Panagiotopoulos, C., Ronsley, R., Al-Dubayee, M., Brant, R., Kuzeljevic, B., Rurak, E., \& Masse, L. C. (2011). The Centre for Healthy Weights-Shapedown BC: A Family-Centered, Multidisciplinary Program that Reduces Weight Gain in Obese Children over the Short-Term. International Journal of Environmental Research and Public Health, 8(12), 4662-4678.

Pérez-Lobato, R., Reigal, R.E., \& Hernández-Mendo, A. (2016). Relationships between physical activity, fitness and attention in an 
adolescent sample. Revista de Psicología del Deporte, 25(1), 179186.

Pontifex, M. B., Raine, L. B., Johnson, C. R., Chaddock, L., Voss, M. W., Cohen, N. J., Kramer, A.F., \& Hillman, C. H. (2011). Cardiorespiratory fitness and the flexible modulation of cognitive control in preadolescent children. Journal of Cognitive Neuroscience, 23, 1332-1345

Posner, M. I., \& Petersen, S. E. (1990). The attention system of the human brain. Annual review of neuroscience, 13(1), 25-42.

Posner, M. I., Rothbart, M. K., Sheese, B. E., \& Voelker, P. (2014). Developing attention: behavioral and brain mechanisms. Advances in Neuroscience, 2014, 1-9.

Rasberry, C.N., Lee, S.M., Robin, L., Laris, B.A., Russell, L.A., Coyle, K.K., \& Nihiser, A.J. (2011). The association between schoolbased physical activity, including physical education, and academic performance: A systematic review of the literature. Prev Med, 52(S10-S20).

Real Decreto 561/1993, de 16 de abril, por el que se establecen los requisitos para la realización de ensayos clínicos con medicamentos. B.O.E. n. ${ }^{\circ} 114$, de 13 de mayo de 1993. Madrid: Ministerio de Sanidad y Consumo.

Recomendaciones de Buena Práctica Clínica de la CEE (1990). Documento 111/3976/88 de julio de 1990.

Repáraz, C., Peralta, F., \& Narbona, J.(1996). El Test de Percepción de Diferencias (CARAS) como instrumento de medida de la atención sostenida. Revista de Ciencias de la Educación, 166, 265-280.

Rowland, T.W. (2007). Evolution of maximal oxygen uptake in children. Tomkinson, G.R. y Ods, T.S. (Eds.), In Pediatric Fitness Secular trends and Geographic Variabiliy (pp. 200-209). Suiza: Karger Publishers.

Ruiz, J. R., España Romero, V., Castro Piñero, J., Artero, E. G., Ortega, F. B., Cuenca García, M. Jiménez-Pavón, D., Chillón, P., GirelaRejón, M.a J., Mora, J., Gutiérrez, A., Suni, J., Sjöstrom, M., \& Castillo, M. J. (2011). Batería ALPHA-Fitness: test de campo para la evaluación de la condición física relacionada con la salud en niños y adolescentes. Nutrición Hospitalaria, 26(6), 1210-1214.

Ruiz, J. R., \& Ortega, F. B. (2009). Physical activity and cardiovascular disease risk factors in children and adolescents. Curr Cardiov Risk Rep., 3(4), 281-287.

Sardinha, L. B., Marques, A., Martins, S., Palmeira, A., \& Minderico, C. (2014). Fitness, fatness, and academic performance in seventhgrade elementary school students. BMC pediatrics, 14, 176.

Schmidt, M., Bezing, V., \& Kamer, M. (2016). Classroom-Based Physical Activity Breaks and Children's Attention: Cognitive Engagement Works! Frontiers in Psychology, 4(7), 1474.

Scudder, MR., Federmeier, K. D., Raine, L. B., Direito, A., \& Boyd, J. K. (2014). The association between aerobic fitness and language processing in children: Implications for academic achievement. Brain and Cognition, 87, 140-152.

Sibley, B.A., \& Etnier, J.L. (2003). The relationship between physical activity and cognition in children: A meta-analysis. Pediatr Exerc Sci, 15,243-56.

Sloan, R.A., Sawada, S.S., Martin, C.K. ,Church, T., \& Blair S.N. (2009). Associations between cardiorespiratory fitness and healthrelated quality of life. Health Qual Life Outcomes, 7, 47.

Sui, X., Laditka, J.N., Church, T.S., Hardin, J.W., Chase, N., Davis, K., \& Blair, S.N. (2009). Prospective study of cardiorespiratory fitness and depressive symptoms in women and men. $J$ Psychiatr Res, 43(5), 546-52.

Tang, Y. Y., Hölzel, B. K., \& Posner, M. I. (2015). The neuroscience of mindfulness meditation. Nature Reviews. Neuroscience, 16(4), 213.

Tang, Y., \& Posner, M. I. (2009). Attention training and attention state training. Trends in Cognitive Sciences, 13, 222-227.

Thomas, J. R., \& Nelson, J. K. (2007). Métodos de investigación en actividad física. Barcelona: Paidotribo.

Thurstone, L. L. \& Yela, M. (2012). Caras-R: test de percepción de diferencias-revisado: manual. Madrid: Tea.

Tomporowski, P., Davis, C., Miller, P., \& Naglieri, J. (2008). Exercise and Children's Intelligence, Cognition, and Academic Achievement. Educational Psychology Review, 20, 111-131.

Tomporowski, P.D., Lambourne, K., \& Okumura, M.S.(2011). Physical activity interventions and children's mental function:An introduction and overview. Preventive Medicine, 52(Suppl 1), S3-S9.

Torres-Luque, G, Carpio, E., Lara, A., \& Zagalaz, M.L. (2014). Fitness levels of elementary school children in relation to gender and level of physical activity. Retos. Nuevas tendencias en Educación Física, Deporte y Recreación, 25, 17-22.

Trudeau, F., \& Shephard, R.J. (2008). Physical education, school physical activity, school sports and academic performance. Int J Behav Nutr Phys Act., 5, 10.

Verburgh, L., Königs, M., Scherder, E. J., \& Oosterlaan, J. (2013). Physical exercise and executive functions in preadolescent children, adolescents and young adults: a meta-analysis. Br J Sports Med, bjsports-2012.

Wass, S., Porayska-Pomsta, K., \& Johnson, M. H. (2011). Training attentional control in infancy. Curr Biol., 21(18), 1543-7.

Wilson, A., Olds, T., Lushington, K., Petkov, J., \& Dollman, J. (2016). The impact of 10-minute activity breaks outside the classroom on male students' on-task behaviour and sustained attention: a randomised crossover design. Acta Paediatrica, 105(4):e181-188.

Zurita-Ortega, F., Ubago-Jiménez, J.L., Puertas-Molero, P., GonzálezValero, G., Castro-Sánchez, M., \& Chacón-Cuberos, R. (2018). Physical activity levels of Primary Education students in Granada. Retos, 34, 218-221
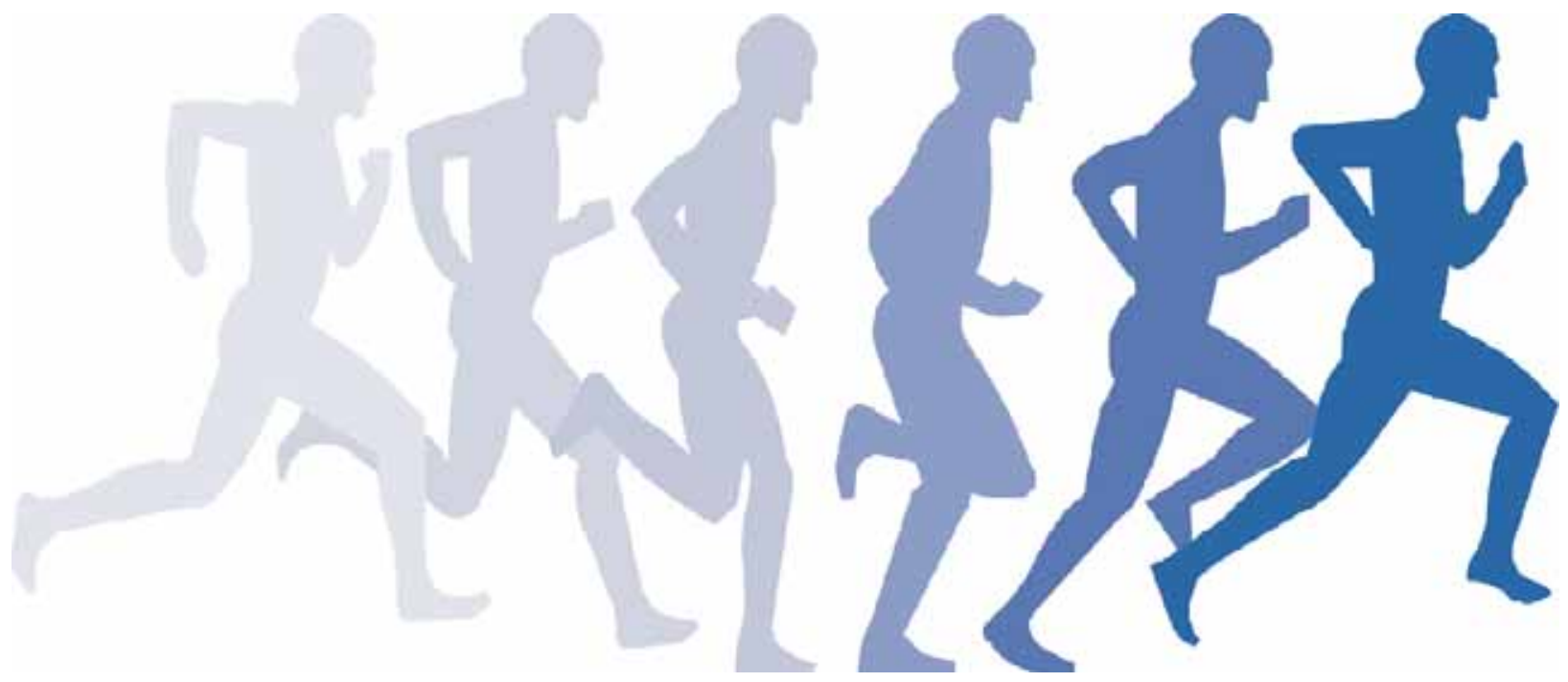